\title{
Energy deposition in the ionosphere through a global field line resonance
}

\author{
I. J. Rae, C. E. J. Watt, F. R. Fenrich, I. R. Mann, L. G. Ozeke, and A. Kale \\ Department of Physics, University of Alberta, Edmonton, Alberta, T6G 2G7, Canada \\ Received: 11 January 2007 - Revised: 5 Sepember 2007 - Accepted: 16 November 2007 - Published: 2 January 2008
}

\begin{abstract}
We present an interval whereby we can estimate the energy dissipation in the ionosphere through an externally-driven field line resonance. In this paper, we utilise an interval described in general by Rae et al. (2005), where the global magnetospheric cavity was shown to be energised via a high solar wind speed stream. Using the ground-based instrumentation available, we estimate the spatial extent of the generated pulsations to be at least $10^{\circ}$ in latitude and $65^{\circ}$ in longitude, a sizeable fraction of the dusksector ionosphere. Using a fortuitous conjunction with the Polar spacecraft, we compare point measurements of the net downward Poynting vector to the estimated Joule heating rate in the ionosphere, and find that model values of the Pedersen conductance are reasonable. In the interval of interest, we estimate the total dissipation rate during a global field line resonance to be comparable to that reported in substorm studies. Previous studies have estimated the total energy deposition via field line resonance to be up to $4 \%$ of that deposited during a small substorm. However, in this paper we find that the total energy deposited via Joule heating may actually be $30 \%$ or more of the energy deposited in the ionosphere during a substorm cycle using a conservative estimate of the pulsation duration.+
\end{abstract}

Keywords. Ionosphere (Electric fields and currents) Magnetospheric physics (Magnetosphere-ionosphere interactions; Solar wind-magnetosphere interactions)

\section{Introduction}

Solar wind energy, mass and momentum can be transferred into the near-Earth environment via both direct and indirect physical processes. Magnetic reconnection (e.g., Dungey, 1961) permits direct access of the solar wind into the Earth's

Correspondence to: I. J. Rae

(jrae@phys.ualberta.ca) magnetosphere and ionosphere where the opening of flux through dayside reconnection ultimately results in the closure of flux on the nightside. The return of this closed flux towards the dayside magnetopause completes the Dungey cycle of magnetospheric convection, and also results in the completion of the auroral oval, tracing out an enormous region of the ionosphere which solar wind and magnetospheric plasma can permeate. On the other hand, indirect processes tend to focus on Ultra Low Frequency (ULF) perturbations. Standing mode ULF oscillations on magnetospheric field lines were first postulated by Dungey (1955), and since then the magnetospheric field line resonance (FLR) has been studied extensively, both theoretically (e.g., Southwood, 1974; Chen and Hasegawa, 1974) and observationally (e.g., Samson et al., 1971; Greenwald and Walker, 1980; Allan et al., 1986). Standing ULF wave modes can be separated into two categories; those with excitation mechanisms internal or external to the magnetosphere. Internal excitation mechanisms can involve resonance with energetic particle sources (e.g., Southwood et al., 1969), or coupling to fundamental plasma instabilities such as the drift-mirror mode (e.g., Pokhotelov et al., 1985, 1986; Woch et al., 1988; Rae et al., 2007). External FLR sources have been shown to be related to either solar wind discontinuity driven cavity/waveguide modes (e.g., Allan et al., 1986; Wright, 1994) or the Kelvin-Helmholtz instability on the magnetopause flanks (e.g., Southwood, 1974; Chen and Hasegawa, 1974; Kivelson and Southwood, 1985, 1986; Mann et al., 2002; Rae et al., 2005 - herein after referred to as R05). Many of these studies invoked a form of the magnetospheric waveguide in order to explain their results, where the magnetopause forms the outer boundary of the waveguide, and a strong gradient in the Alfvén speed forms the inner boundary for the compressional mode waves. Compressional wave energy may then excite FLRs where the frequency of the compressional waves matches the local standing mode FLR field line eigenfrequency. The ultimate sink for this extracted solar wind energy is the ionosphere,

Published by Copernicus Publications on behalf of the European Geosciences Union. 
where it is deposited via energetic auroral particles or Joule heating.

To study the global nature of the magnetosphereionosphere energy sink due to energetic auroral particles requires remote sensing via Global Imagers, typically from the Polar and IMAGE spacecraft (e.g., Fillingim et al., 2002; Østgaard et al., 2002a, b). Keiling et al. (2003) used the Polar spacecraft to simultaneously image the nightside northern hemisphere ionosphere with the UVI instrument whilst the spacecraft was conjugately located in the Plasmasheet Boundary Layer (PSBL). In their work, Keiling et al. (2003) measured both the in-situ Poynting vector and the ionospheric electron energy flux associated with discrete auroral forms, and found that there was a high correlation between intense Poynting vector and auroral emission intensity. Furthermore, Keiling et al. (2003) showed that the magnetospheric in-situ Poynting vector mapped into the ionosphere was 1-10 times larger than the ionospheric electron energy flux, confirming that Alfvénic perturbations in the PSBL can have more than enough energy to produce auroral forms. Standing Alfvén waves, or FLRs, are thought to be an important contributor to the acceleration of auroral electrons in some types of discrete auroral arcs (e.g., Hasegawa, 1976; Greenwald and Walker, 1980; Allan and Poulter, 1984; Samson et al., 1991, 1996; Milan et al., 1999, 2001).

Few studies have attempted to quantify the total energy deposition in the auroral oval, either via direct or indirect solar wind excitation. Østgaard et al. (2002b - herein after referred to as $\varnothing 02 \mathrm{~b}$ ) presented several case studies whereby an estimate of the total energy budget of a substorm could be estimated from global auroral imaging. In their study, Ø02b. found that the total energy budget during a substorm was $10^{15}-10^{16} \mathrm{~J}$ and that energy dissipated into the ionosphere via Joule heating may be $40-60 \%$ of those values. Interestingly enough, Ø02b found the energy dissipation via auroral electrons were 2-4 times larger than previously estimated during the substorm cycle. It is important to note that the total energy depositions discussed in Østgaard et al. (2002a, b) are not entirely equivalent to the studies presented here, since the substorm cycles analysed by these authors had durations of 4-11 h. They are, however, included and discussed in Sect. 4 for comparison.

Fewer still have studied energy deposition via ULF waves or a field line resonance, presumably because of its inherent difficulties; rarely is there sufficient observational coverage to attempt even a rough calculation. Dessler (1959) estimated that shorter period Pc1 $(0.2-5 \mathrm{~s}$ period - Jacobs et al., 1964) waves dissipated $\sim 10^{11} \mathrm{~J}$ into the ionosphere during the lifetime of the resonance. Greenwald and Walker (1980, herein after referred to as GW80) were the first authors to quantify the dissipation from longer period Pc5 (150-600 s period) FLRs. In their paper, GW80 used VHF (Very High Frequency) radar measurements of the E-region ionosphere from the STARE (Scandinavian Twin Auroral Radar Experiment) radar to study both the energy stored in toroidal mode FLRs and the energy dissipation in the ionosphere from those perturbations via Joule Heating, and found them to be $\sim 10^{13} \mathrm{~J}$ and $6 \times 10^{9} \mathrm{~W}$, respectively. As an interesting aside, GW80 were the first authors to predict that the energy deposition through these resonant waves may be enough to excite poleward-moving bands of auroral precipitation, a feature validated and so closely studied in recent times.

Gla $\beta$ meier et al. (1984) studied a sudden impulse driven Pc5 pulsation to establish that the dominant damping mechanism for ULF pulsations was via ionospheric Joule heating, where the rate of damping was proportional to both the Pedersen conductance and the L-shell of the observation (e.g., Newton et al., 1978). A more continuous series of ULF pulsations must therefore extract sufficient energy from its source mechanism in order to balance the Joule dissipation (e.g., Hughes and Southwood, 1976).

Allan and Poulter (1984 - herein after referred to as AP84) reported Pc5 FLR activity may deposit $10^{10}-10^{13} \mathrm{~J}$ of energy into the ionosphere, depending on whether the pulsations were wave-particle driven (polodially-polarised), or externally excited (toroidally-polarised). Baddeley et al. (2005 - herein after referred to as B05) studied cases for which the Joule heating could be estimated during two classes of high$m$ FLRs that were driven via energetic particle resonance. In their study, B05 used a method similar to GW80 - and detailed in Sect. 3 of this paper - to estimate that a high-m resonance could deposit $10^{10}-10^{11} \mathrm{~J}$ into the ionosphere through Joule heating, representing a significant energy sink in the magnetosphere-ionosphere system.

Buchert et al. (1999) utilised incoherent scatter radar and magnetometer measurements to characterise the modulation of particle precipitation and ionospheric conductivities via poloidal Pc5 FLR. In their paper, Buchert et al. (1999) determined that ionospheric conductivities varied by a factor of $\sim 2$ in the presence of ULF pulsations. These authors concluded that these variations in conductivities were due to the periodic modulation of electrons into the loss cone, at least in their case study. In the same manner, Rae et al. (2007) presented an interval during which there were contemporaneous observations of optical, magnetic and riometer absorption signatures of a two discrete toroidal FLRs in the dawn sector. In their paper, these authors linked the magnetic signatures of the FLR wave fields with both the soft and hard electron precipitation in that region. These authors also pointed to the modulation of VLF growth rates by compressional ULF waves as the likely candidate to scatter these electrons into the loss cone (e.g., Coroniti and Kennell, 1970).

R05 presented an interval of high solar wind speed, during which a remarkable array of instrumentation observed externally-driven Pc5 ULF wave activity from the dusk-flank magnetopause, through the magnetosphere and into the ionosphere. In their paper, Rae et al. focussed on a three hour subset of the interval whereby these authors traced solar wind energy from the magnetopause undulations through the outer magnetosphere, and into the ionosphere. This event 


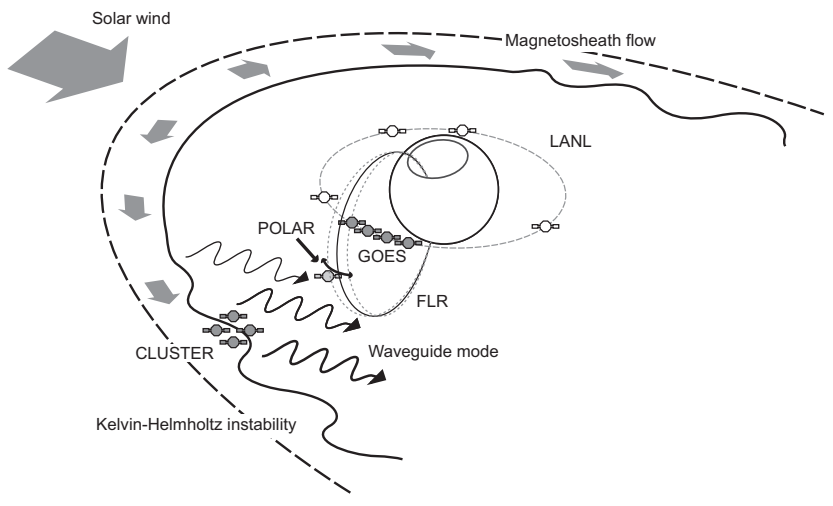

Fig. 1. A schematic representation of the results reported in R05. This schematic is shown from the post-dusk sector above the equatorial plane, with the sun and solar wind in the top left hand corner, and the magnetotail stretching in the bottom right direction.

is consistent with Kelvin-Helmholtz excitation at the magnetopause and excitation of the magnetospheric waveguide, perhaps via the over-reflection mechanism (see Mann et al., 1999). In this paper we present an extension of the event reported in R05 in order to quantify the energy deposition into the ionosphere, and compare the resultant Joule heating with the in-situ Poynting vector observed directly conjugate to the FLR region in order to study the energy budget during resonance. Through this study, we can reasonably estimate both point measurements of the Pedersen conductance, and the energy deposited into the ionosphere, and quantitatively estimate the energies associated with the periodic auroral forms postulated by GW80 and studied by many others in the intervening years.

\section{Methodology}

\subsection{Event history}

Figure 1 shows a schematic representation of the conclusions reported in R05. In this paper, the authors determined that a high solar wind speed event $\left(v_{s w}>700 \mathrm{kms}^{-1}\right)$ may have excited the magnetospheric waveguide, and driven monochromatic compressional waves into the cavity, thus exciting FLRs in the vicinity of the Polar spacecraft. During the interval, the Cluster spacecraft were traversing the dusk-side magnetopause, and observed monochromatic fluctuations in magnetic field strength, ion spectra, and derived ion velocities (Fig. 4, R05) characteristic of Kelvin-Helmholtz excitation of the magnetopause. The Polar spacecraft was situated in the outer magnetosphere between $9-11 R_{E}$ in approximate radial alignment with the Cluster constellation and observed monochromatic radial electric and azimuthal magnetic field variations which were clearly $90^{\circ}$ out of phase and as expected for a standing Alfvén wave. Geosynchronous observations from the GOES and LANL spacecraft constel-

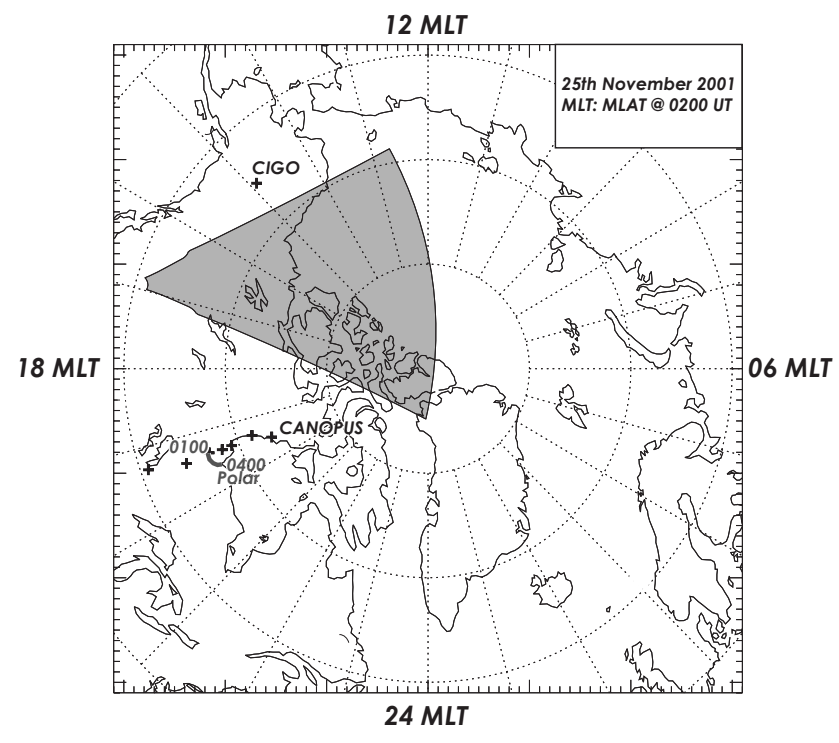

Fig. 2. The ionospheric footprint of the relevant instrumentation on the 25 November 2001 at 02:00 UT in AACGM MLT: MLAT coordinates looking down on the northern hemisphere with noon at the top of the figure. Shown in grey is the field-of-view of the Prince George SuperDARN radar, in grey the ionospheric footprint of the Polar (01:00-04:00 UT), the black crosses denote the positions of the CANOPUS (now CARISMA) "Churchill line" magnetometers, and the triangle represents the CGO magnetometer utilised in this study.

lations showed that monochromatic magnetic perturbations were excited in the dusk sector, and that $\sim 100-500 \mathrm{keV}$ electron fluxes were modulated at the same frequency at all local times. Finally, the resonant behaviour was observed on the ground in an extended region around dusk with multiple radars of the SuperDARN (Super Dual Auroral Radar Network - Greenwald et al., 1995) HF radar network and the CANOPUS (Canadian Auroral Network for the OPEN Program Unified Study - see Rostoker et al., 1995 for details) magnetometer chain, since upgraded and extended and is now known as CARISMA (Canadian Array for Realtime Investigations of Magnetic Activity). These ground-based measurements of monochromatic ionospheric flows and ground magnetic perturbations covered a sizeable fraction of the dusk-sector ionosphere, between $\sim 15: 00$ 20:00 MLT (Magnetic Local Time).

\subsection{Instrument location}

The location of the ground-based and ionospheric instrumentation used in this study is shown in Fig. 2. This ionospheric projection is shown looking down on the northern hemisphere ionosphere at 02:00 UT on the 25 November 2001 in magnetic latitude: magnetic local time coordinates (MLT: MLAT); the field-of-view of the SuperDARN Prince George radar is shaded in grey, and the "Churchill Line" of 
$25^{\text {th }}$ Nov 2001: Polar, CARISMA, GIMA and SuperDARN

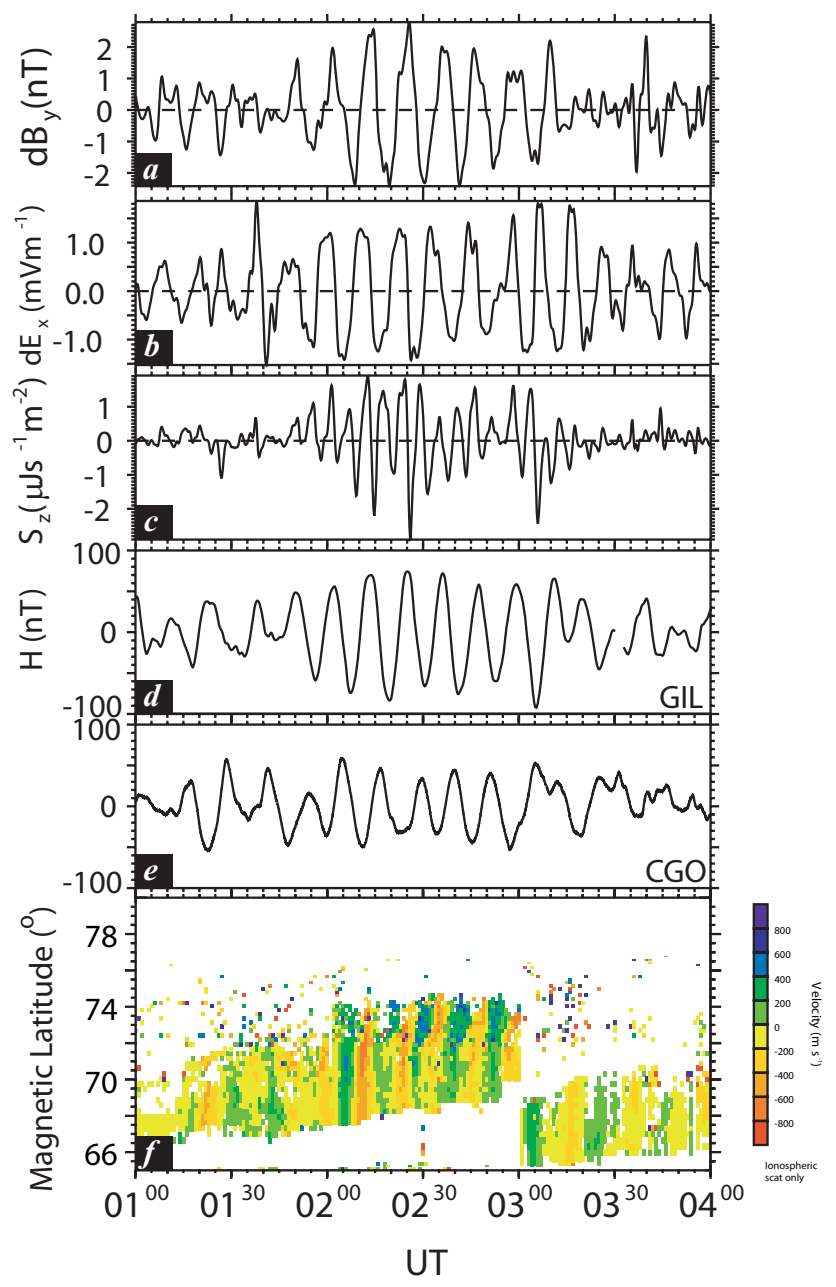

Fig. 3. (a), (b) the in-situ $\sim$ azimuthal and $\sim$ radial magnetic and electric fields, respectively, as measured by the Polar spacecraft, and using the field-aligned co-ordinate system outlined in R05. (c) shows the in-situ field-aligned Poynting vector, (d) the $\mathrm{H}$-component ground magnetometer data from GIL, (e) the $\mathrm{H}$ component ground magnetometer from CGO, and (f) the 1-o-s velocity from beam 5 of the Prince George HF radar. These datasets have been plotted between 01:00-04:00 UT on the 25 November 2001.

the CARISMA magnetometer array is denoted by the black crosses. The ionospheric footprint of Polar is also shown between 01:00-03:00 UT by the grey line. Finally, data from the College International Geophysical Observatory magnetometer (CGO), run by the Geophysical Institute of the University of Alaska Fairbanks is also included in this study, and denoted by a black triangle.

\subsection{Ground-based and ionospheric observations}

Figure 3 shows an overview of the measurements taken on the 25 November 2001 between 01:00-04:00 UT from the Polar spacecraft, and conjugate ground-based and ionospheric measurements, reproduced here as a summary of the key measurements reported in R05 Figs. 5, 8, and 9. In this interval, Polar was traversing the dusk magnetosphere close to the equatorial plane at radial distances of $L \sim 9-11$. From top to bottom, Fig. 3 shows in-situ (a) quasi-azimuthal magnetic field, (b) quasi-radial electric field perturbation, and (c) field-aligned Poynting vector measured by Polar. The data have been band-pass filtered between 1 and $10 \mathrm{mHz}$. The field-aligned co-ordinate system is described in detail in R05; essentially, the use of a field-aligned co-ordinate system allows the determination of the polarisation of the FLR in the equatorial plane, and the net energy transfer via this resonance Fig. 3d and e shows the unfiltered $\mathrm{H}$-component ground magnetometer perturbations from the Gillam (GIL) and CGO magnetometers respectively. Figure $3 \mathrm{f}$ shows a latitude-time plot of line-of-sight-plasma velocity from beam 5 of the Prince George radar where red (blue) represents plasma velocity away from (towards) the radar.

R05 showed that the Polar observations were consistent with a toroidal-mode FLR; the radial electric $\left(d E_{x}\right)$ and azimuthal magnetic $\left(d B_{y}\right)$ fields being clearly $90^{\circ}$ out of phase. In the equatorial plane, the net field-aligned component of the Poynting vector should be $\sim 0$. R05 showed that the net field-aligned Poynting vector was $\sim 0$, but slightly in the positive field-aligned direction i.e. directed away from the equator and towards the northern hemispheric ionosphere, consistent with Polar's magnetospheric location just above the equatorial plane, and therefore close to the magnetic node of the FLR. An integration of the field-aligned Poynting vector over the interval 01:30-03:00 UT gives a net value of $\sim 0.3 \mu \mathrm{Js}^{-1} \mathrm{~m}^{-2}$ towards the northern hemispheric ionosphere.

Figures 3d-e show the extent of the monochromatic perturbations observed on the ground and in the ionosphere. Figure $3 \mathrm{~d}$ shows the H-component measurements from GIL, which is the location of maximum wave amplitude observed on the ground and conjugate to the Polar measurements, and shows clearly the large-amplitude $(150 \mathrm{nT}$ peakto-peak) $1.4-1.6 \mathrm{mHz}$ perturbation in the $\mathrm{H}$-component magnetic field, characteristic of a toroidal mode FLR observed on the ground. The reader is directed to Fig. 8 in R05 for a full description of the ground magnetic perturbations. Figure $3 \mathrm{e}$ shows the $\mathrm{H}$-component magnetometer trace from the CGO magnetometer, around the same magnetic latitude as GIL but separated by $\sim 4 \mathrm{~h}$ of local time. Again, a clear largeamplitude (140 nT peak-to-peak) monochromatic perturbation can be seen. There is a small associated D-component perturbation ( $20 \mathrm{nT}$ peak-to-peak, not shown), characteristic of a toroidal mode FLR observed on the ground. Figure $3 \mathrm{f}$ shows the F-region plasma velocity is also perturbed by these 
ULF wave fields at the same frequency from Prince George beam 5 , some $4 \mathrm{~h}$ of local time away, and in the post-noon ionosphere. Again, we direct the reader to R05 Fig. 9 for the entirety of the plasma velocity measurements; beam 5 of the Prince George radar is taken as representative of these measurements. This line-of-sight (1-o-s) plasma velocity is perturbed by up to $\pm 700 \mathrm{~ms}^{-1}$ in the interval 02:00-03:00 UT.

In order to identify the longitudinal extent of the resonant signatures during this period, we present amplitude and phase characteristics of the $1.5 \mathrm{mHz}$ ULF wave component of the signals in both the SuperDARN Prince George radar and the CARISMA "Churchill Line" magnetometers. Figure 4 shows the $1.5 \mathrm{mHz}$ (top panels) amplitude and (bottom panels) phase characteristics as a function of latitude from both (a) ionospheric measurements of the Prince George 1-o-s velocity and (b) H-component ground magnetic perturbations from the CARISMA "Churchill line" magnetometers, reproduced from Fig. 8b, R05. Figures $4 \mathrm{a}$ and $\mathrm{b}$ show that there are clear peaks in the amplitude of the $1.5 \mathrm{mHz}$ component of both the line-of-sight plasma velocity as well as the $\mathrm{H}$ component magnetic field. Together with the $\sim 180^{\circ}$ phase change across this amplitude peak, this is clear evidence that both observations are signatures of a toroidal mode FLR (e.g. Walker et al., 1979; Samson et al., 1992; Fenrich and Samson, 1997) that are separated by $60^{\circ}$ in longitude.

In the following section, we present analysis of these observations, and their relationship to established theory.

\section{Energy budgets}

\subsection{Joule heating in the ionosphere}

Hughes and Southwood (1976) showed that the shielding of the ULF waves by Pedersen currents means that in the ionosphere, the magnetic field perturbation is:

$b=\mu_{0} \Sigma_{p} E$,

where $\mu_{0}, \Sigma_{p}, E$ are the permittivity of free space, the Pedersen conductivity and the perpendicular root mean squared ionospheric electric field, respectively.

Rewriting this equation, we see that the net downward Poynting vector may be balanced by the Joule heating via Pedersen currents in the ionosphere:

$$
\frac{E b}{\mu_{0}}=\Sigma_{p} E^{2} .
$$

Integrating the local dissipation rate on the right hand side of Eq. (2) with respect to the approximate area over which this deposition takes place, we find the total dissipation rate:

$D=\int \Sigma_{p} E^{2} d A$.

In this paper, we assume that $E$ varies only with latitude. We justify this assumption in several stages. Firstly, there

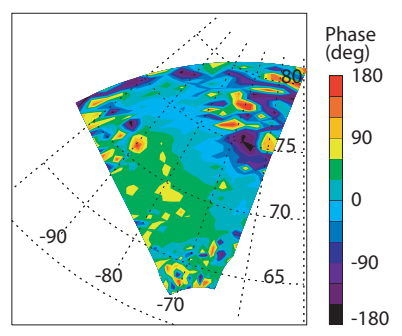

(a) SuperDARN Prince George

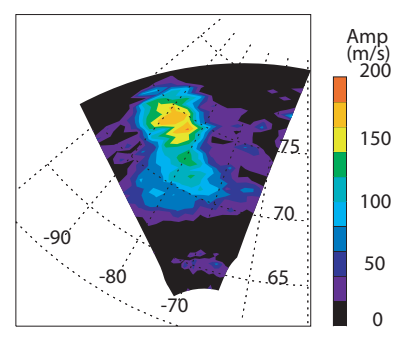

(b) CARISMA "Churchill" Line
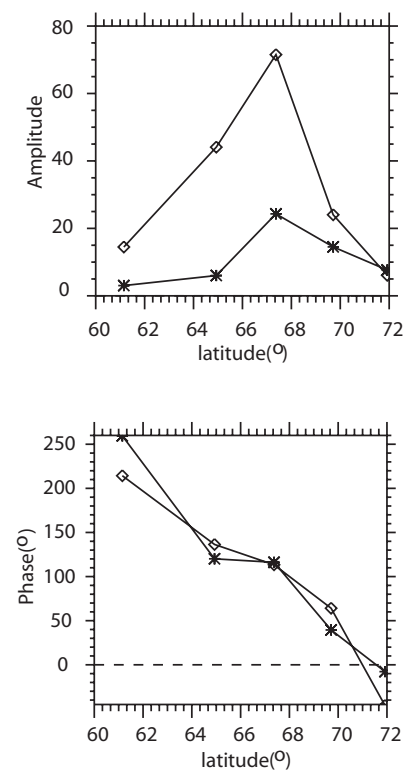

Fig. 4. (top) the amplitude and (bottom) phase of the $1.5 \mathrm{mHz}$ components of (a) the SuperDARN Prince George radar 1-o-s velocities, and (b) the H- (diamonds) and D- (stars) component magnetometer data from the Churchill line meridian.

are no $E$ measurements spanning the measurements taken in the local time sectors between the 17:00-19:00 MLT regions shown in Fig. 2. Secondly, using Eq. (1) we can estimate the expected electric field conjugate to the ground magnetometer measurements. Using the requisite $\Sigma_{p}$, and employing the technique of Hughes and Southwood (1976) and Gla $\beta$ meier et al. (1984) to map the ground $\mathrm{b}$ into the ionosphere, we find that the ionospheric electric field above the CGO magnetometer was $\sim 20 \mathrm{mVm}^{-1}$, which is similar to the observations of the electric fields observed in the near range-gates of beam 5 of the Prince George radar. In practice it is difficult to measure $\Sigma_{p}$ in the ionosphere since this requires suitable instrumentation conjugate to the area of interest. However, model values can be used, such as the IRI-90 (International Reference Ionosphere - Bilitza, 1990) model. Typical daytime conductances are greater than $1 \mathrm{~S}$, typical nightside conducances are an order of magnitude lower. It therefore becomes impractical to assume that $\Sigma_{p}$ close to the dawn-dusk terminator on the flanks will be a constant, and so we must estimate $\Sigma_{p}$ for the area of deposition. Table 1 shows the variation of $\Sigma_{p}$ with local time at 02:30 UT according to the IRI-90 model. The Pedersen conductance varies with local time between $\sim 0.5-3 \mathrm{~S}$. We observe the FLR signature over at least $65^{\circ}$ of longitude, and $10^{\circ}$ of latitude which is bounded by 15:00-20:00 MLT and 66.5-76.5 MLAT. By numerically integrating these values over the section of spherical area defined by the ULF wave activity and the ionosphere, we can estimate the average $\Sigma_{p}$ for the FLR 


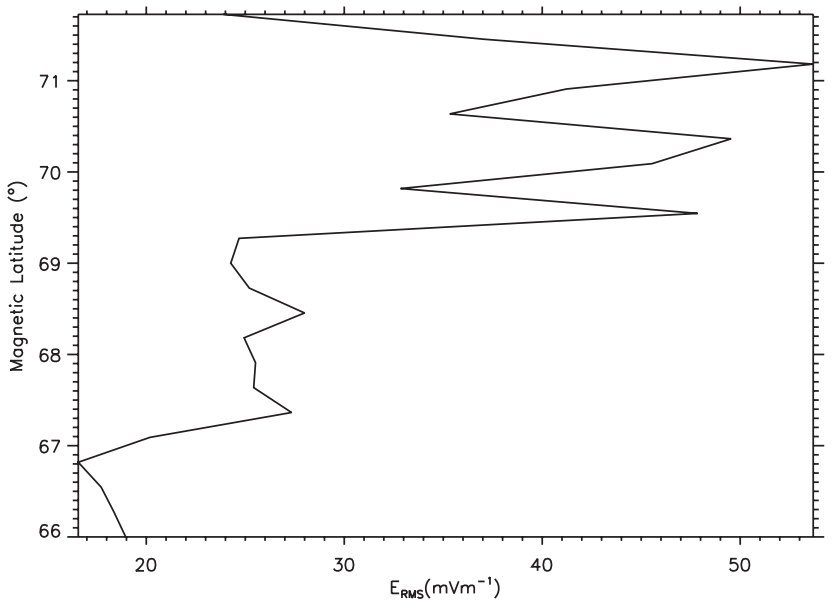

Fig. 5. The root mean squared electric field magnitude observed in Prince George beam 5 during the interval 02:00-03:00 UT as a function of magnetic latitude.

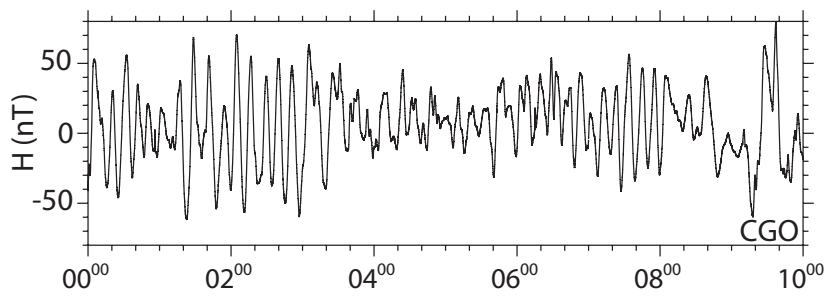

Fig. 6. The H-component ground magnetometer data from CGO for the interval 00:00-10:00 UT on the 25 November 2001.

region which spans the dusk terminator. The average conductance in this region is $\Sigma_{p}=1.080 \mathrm{~S}$; although we use the range of point estimates given in Table 1 in our integration, this value is useful to compare with the estimates of $\Sigma_{p}$ discussed in Section 4. The Pedersen conductance will obviously have a large effect on the local time variation of the electric field. However, using the technique of Hughes and Southwood (1976) and Glaßmeier et al. (1984) again, we find that a similar amplitude FLR at GIL $\left(\sim 66^{\circ}\right)$ at $\sim 19: 00$ MLT will have $E_{\text {MAX }} \sim 80 \mathrm{mVm}^{-1}$, a factor of 4 larger than the same latitude at $\sim 15: 00$ MLT due to the differing $\Sigma_{p}$. Due to the lack of longitudinal coverage, we simply argue that we do not have the instrumental coverage to resolve this problem, but use the fact that the electric fields derived at GIL are close to that observed by the SuperDARN Prince George radar at the same corresponding latitude. Therefore we assume that the electric field does not vary with longitude. Indeed, the estimates of the variation of $E$ above indicate that our calculations of dissipative power and energy may be $\sim 2$ times larger.

The damping rate of a guided Alfvén wave is dependent on $\Sigma_{p}$ at both ends of the field line (Ozeke et al., 2005), however, we do not have any information in the conjugate hemi-
Table 1. The ionospheric Pedersen conductances estimated from the IRI-90 model (Bilitza, 1990) for the four hours of local time that the field line resonance encompasses.

\begin{tabular}{lll}
\hline Local Time (MLT) & $\left(\Sigma_{p}\right)_{66.5^{\circ}}$ & $\left(\Sigma_{p}\right)_{76.5^{\circ}}$ \\
\hline 15 & 3.0 & 1.1 \\
16 & 2.0 & 0.93 \\
17 & 1.3 & 0.79 \\
18 & 0.96 & 0.67 \\
19 & 0.72 & 0.57 \\
\hline
\end{tabular}

sphere. Instead, we make the simple assumption that over such an extended region of the ionosphere, the Joule dissipation of ionospheric currents would be similar in both resistive ionospheres, though we caution that this may not necessarily be the case, we do not have the instrumental coverage to verify this.

Ideal MHD allows us to calculate an estimate of the ionospheric electric field from the line-of-sight plasma velocity, or at least a lower boundary on this value given that we do not have sufficient conjugate radar measurements to estimate a 2-D plasma velocity. Figure 5 shows the maximum electric field magnitude observed in beam 5 during the interval 02:00-03:00 UT as a function of magnetic latitude. We take this latitudinal profile of the electric field as representative of the entire interval.

Using Eq. (2) and the measurements and estimates detailed above, the total dissipation rate is found to be $D=9.9 \times 10^{9} \mathrm{~W}$. We assume that the energy of a global mode will be deposited in both the northern and southern hemispheres, and will most likely be deposited on both the dawn and dusk flanks. Although no conjugate measurements could be obtained to verify this assumption from the southern hemisphere, the Greenland and IMAGE magnetometers observed $\sim 100 \mathrm{nT}$ peakto-peak Pc5 ULF oscillations closer to the dawn flank (not shown). We can therefore estimate the total energy dissipated, $E_{\mathrm{TOT}}=4 \mathrm{DT}$, where $T$ is the interval length.

Obviously, the duration of the interval is of critical importance in the estimation of the total energy dissipated during field line resonance. R05 concentrated on a subset of the entire ULF wave interval, but their Fig. 9 showed that the ionospheric velocity perturbations were observed in an $\sim 8-12 \mathrm{~h}$ UT interval by the SuperDARN radars as the ground stations rotated through the dusk sector ( 15:00-20:00 MLT). For example, Fig. 9f of R05 shows that the Iceland West radar observed ULF wave activity from 20:00 UT on the 24 November 2001, though of a slightly different frequency. It is difficult to determine that the entirety of these ULF perturbations were resonant. However, using the CGO magnetometer in Alaska, we can show that there were large-amplitude magnetic perturbations of the same frequency $(1.4-1.6 \mathrm{mHz})$ over the interval $\sim 00: 00-08: 00$ UT. Figure 6 shows the H- 
component magnetic trace from the CGO magnetometer site, situated at $65.2^{\circ}$ and $264.6^{\circ}$ magnetic latitude and longitude, respectively. It is evident that there are large-amplitude $\mathrm{H}$ component magnetic perturbations (up to $140 \mathrm{nT}$ peak-topeak) observed by the CGO magnetometer over the entire 8-h period. In this paper, we conservatively estimate of the duration of the pulsations, since Fig. 9a, b in R05 demonstrate that there is ULF wave activity observed for at least the 00:00-04:00 UT interval. However, since we cannot verify the latitudinal and longitudinal extent of the pulsations as rigorously in the later interval (03:00-08:00 UT), we conservatively estimate the pulsation duration to be three hours, spanning 01:00-04:00 UT. In this three hour period, we estimate the total energy dissipated in the ionosphere to be $E_{\mathrm{TOT}}=4.26 \times 10^{14} \mathrm{~J}$. Of course, if the pulsations shown in Fig. 6 do indeed represent a discrete frequency FLR of 8-h duration, then we should adjust this total accordingly, and the total energy deposition in the ionosphere may be $E_{\mathrm{TOT}}=1.14 \times 10^{15} \mathrm{~J}$.

\subsection{Ground-satellite conjugate measurements}

Since the Polar spacecraft is traversing the resonant region, conjugate to the ground magnetic perturbations, we can estimate the accuracy of the IRI-90 model $\Sigma_{p}$ estimates through comparison of the local dissipation rate to the net downward Poynting vector. Assuming that magnetic flux is conserved along a geomagnetic field line, the cross-sectional area of that flux tube is inversely proportional to the local magnetic field strength. Polar was situated close to the equatorial plane, and observed a local magnetic field strength that varied between 40-46nT (not shown). The magnetic field strength conjugate to Polar on the ground was $\sim 61100 \mathrm{nT}$, and so the corresponding flux tube would have a crosssectional area 1330-1530 times smaller on the ground. In the interval 01:30-03:00 UT, the average net downward Poynting vector is calculated to be $0.3 \mu \mathrm{Js}^{-1} \mathrm{~m}^{-2}$, which corresponds to a net Poynting vector of $400-460 \mu \mathrm{Js}^{-1} \mathrm{~m}^{-2}$ at the conjugate point on the ground.

Hughes and Southwood (1976) showed that the attenuation of transverse electric fields is minimal throughout the lower ionosphere and atmosphere (see their Fig. 4). Since the perpendicular electric fields do not vary vertically on scales of $\sim 10^{2} \mathrm{~km}$, we assume that the electric fields observed by SuperDARN are representative of the entire ionospheric electric field structure.

To compare this net downward Poynting vector to the Joule heating in the ionosphere, we use the Pedersen conductance $\Sigma_{p} \sim 0.5 \mathrm{~S}$ from the IRI-90 model at the mapped ionospheric location of the spacecraft at 02:30 UT, rather than the average $\Sigma_{p}$ calculated earlier. We therefore calculate the local dissipation rate as $475 \mu \mathrm{Js}^{-1} \mathrm{~m}^{-2}$, which is remarkably close to the net downward Poynting vector $S_{z}=400$ $460 \mu \mathrm{Js}^{-1} \mathrm{~m}^{-2}$ measured by Polar.

\section{Discussion}

The dissipation of energy via Joule heating in the ionosphere is fundamental to the study of the formation of auroral features. It is therefore helpful to estimate both the total energy deposition into the ionosphere, and the rate at which this deposition is taking place. In this paper, we estimate the energy deposition in the ionosphere through a "Global mode" field line resonance, using an interval diagnosed as a KelvinHelmholtz excited magnetospheric waveguide mode by R05.

Few studies have attempted to quantify the energy deposition via field line resonance, Table 2 summarises these previous results. It is important to note that GW80 studied solely toroidal-mode FLRs, AP84 both toroidal and poloidal mode FLRs, and B05 solely poloidal mode FLRs. Since there are relatively few studies with sufficient observations able to estimate this energy budget, we include a study of the energy budget during the substorm cycle by $\varnothing 02 \mathrm{~b}$ in Table 2 . Though a different study, $\varnothing 02 b$. provides reference for a partial energy budget during a substorm and a comparison between the Joule heating rates during a substorm and a global field line resonance. Table 2 shows the local and total dissipation rates, together with the total energy deposited in the ionosphere through Joule heating in the previous studies. Where the studies quoted did not give all three values, in some cases it was possible to deduce values from other information contained in the study. Values are omitted where this was not possible.

It is important to note that each calculation of the energy storage and release discussed in this paper and by $\varnothing 02 b, G W 80$, and B05 are subtly different. GW80 estimated the Joule heating using the root-mean squared maximum electric field value in their desired frequency range (3.2$4.5 \mathrm{mHz}$ ) in the ionosphere to give an estimate of the power of the FLR, and used this value as representative of the entire area of interest. Greenwald and Walker also estimated the latitudinal and longitudinal extent of the pulsations to be $100 \times 3000 \mathrm{~km}\left(\sim 1 \times 30^{\circ}\right)$, respectively, their measurements were confined to a small section of the E-region ionosphere over which the Pc5 pulsation may have been occurring. Finally, a theoretical fit to the data was employed to obtain an average value of $\Sigma_{p}$ over the assumed area, and found to be $\Sigma_{p}=2.5 \mathrm{~S}$. This value is representative of the daylit ionosphere, however, this may be an overestimate of the average conductance value on the flanks close to, or spanning, the dawn-dusk terminator, as we see from the model predictions detailed in Table 1 . In their studies of poloidallydriven FLRs, B05 used the maximum electric field amplitude to estimate the Joule heating present in high-m field line resonance to compare to the free energy available in unstable particle distributions observed at the same L-shells in the magnetosphere by the Polar spacecraft. These authors estimated a dissipation area of $1 \times 5^{\circ}$ in latitude and longitude in the ionosphere, respectively, which was taken as typical for a high- $m$ FLR at $L \sim 6$ (e.g., Baddeley et al., 2002). Therefore, 
Table 2. A comparison of the previous work on energy deposition into the ionosphere via Joule heating. For the AP84 values, we assume that the total energy deposition is an hourly rate (through comparison with the GW80 event 7). For comparison, the work of $\varnothing 02 \mathrm{~b}$ are included, who studied the Joule heating during a substorm cycle.

\begin{tabular}{lllll}
\hline Authors and Event & Event Type & $\begin{array}{l}\text { Local Dissipation } \\
\text { Rate }\left(\mathrm{mW} / \mathrm{m}^{2}\right)\end{array}$ & $\begin{array}{l}\text { Total Dissipation } \\
\text { Rate }(\mathrm{W})\end{array}$ & $\begin{array}{l}\text { Total Energy } \\
\text { Deposition }(\mathrm{J})\end{array}$ \\
\hline GW80 Event 7 & low- $m$ & 4.84 & $6 \times 10^{9}$ & $2 \times 10^{13}$ \\
AP84 Event 2 & low- $m$ & - & $1.7 \times 10^{8}$ & $6 \times 10^{11}$ \\
R05 & low- $m$ & $1.3^{*}$ & $9.9 \times 10^{9}$ & $4.3 \times 10^{14 \mathrm{a}}$ \\
& & & & $1.1 \times 10^{15 \mathrm{~b}}$ \\
AP84 Event 3 & high- $m$ & - & $5.6 \times 10^{7}$ & $2 \times 10^{11}$ \\
AP84 Event 4 & high- $m$ & - & $8.3 \times 10^{6}$ & $3 \times 10^{10}$ \\
B05 Event 1 & high- $m$ & 0.075 & $2.7 \times 10^{6}$ & $\sim 10^{10}$ \\
B05 Event 2 & high- $m$ & 0.68 & $7.9 \times 10^{6}$ & $\sim 10^{11}$ \\
$\varnothing 02 b$ Event 1 & substorm & - & $7 \times 10^{10}$ & $4.2 \times 10^{15}$ \\
$\varnothing 02 b$ Event 2 & substorm & - & $4.7 \times 10^{10}$ & $2.6 \times 10^{15}$ \\
$\varnothing 02 b$ Event 3 & substorm & - & $9 \times 10^{10}$ & $2.3 \times 10^{15}$ \\
$\varnothing 02 b$ Event 4 & substorm & - & $8 \times 10^{10}$ & $6.1 \times 10^{15}$ \\
\hline
\end{tabular}

* average local dissipation rate

a 3-h interval duration

b 8-h interval duration

for comparison, the numbers detailed by B05 must be halved for comparison with the rest of Table 2. This factor makes very little difference to their conclusions, since these authors were interested in providing an order of magnitude calculation. Attempting to discern the deposition throughout an entire substorm cycle, $\varnothing 02 \mathrm{~b}$ estimated the energy deposited in intervals $4-11 \mathrm{~h}$ in duration, rather than the $1-3 \mathrm{~h}$ durations of the FLR events, and so their instantaneous estimates of Joule heating need to be adjusted accordingly for comparison (see Table 2).

1. Low- $m$ vs. high- $m$ FLRs. The only studies that have provided local dissipation rates are GW80, B05 and the study presented in this paper. From these studies, the estimated local dissipation rates for high-m FLRs were an order of magnitude lower than for low- $m$ FLRs, simply because of the higher electric fields associated with large-scale resonance. In this present study, we find that the minimum and maximum local dissipation rates in our event are $0.16 \mathrm{~mW} \mathrm{~m}^{-2}$ and $7.3 \mathrm{~mW} \mathrm{~m}^{-2}$, reflecting both the variation in local conductance as well as the latitudinal variation of electric field amplitude due to the resonance. Total dissipation rates follow this trend, with high- $m$ FLR cases depositing at least 2 orders of magnitude less into the ionosphere than in low-m FLR cases, since both the electric field amplitude and the observed region over which the ULF waves act is much larger in the low-m cases. Finally, it is important to note that the calculation for total energy deposition for high- $m$ waves assumes that the FLR is depositing an equal amount of energy in both hemispheres, whereas for the low-m case it is also assumed that the energy will be deposited in both the dawn and dusk flanks. It is unclear whether Allan and Poulter have applied the same assumptions as stated above, but the synthesis of these studies indicate that the total energy deposition for low- $m$ FLRs is significantly greater than that during high- $m$ FLRs.

2. Low- $m$ vs. low- $m$. It is clear that with an extended region of data coverage from multiple sources and with simple modelling of local ionospheric conductance conditions, the diagnosis of energy deposition due to low-m FLRs can be substantially improved. We have expanded the analysis of Greenwald and Walker by retaining the latitudinal amplitude information of the ionospheric electric field, and using a spatially-varying ionospheric conductance obtained from the IRI-90 model. Due to the multi-instrument coverage of this event, we were also able to more accurately determine the ionospheric region over which the energy deposition is assumed to occur.

3. Low- $m$ vs. substorm. GW80 estimated that a low- $m$ FLR may deposit up to $4 \%$ of the energy that is deposited during a substorm. Ø02b estimates that the total deposition rates during a substorm cycle to be larger than those estimated in this present study, primarily because of the extended region and duration over which a substorm cycle takes place. However, we have conservatively estimated the total energy deposited through a 3-h duration low- $m$ FLR to be $30 \%$ or more of the Joule heating measured during a substorm cycle, which is significantly greater than that estimated by Greenwald and Walker. This demonstrates that low-m FLRs are an important consideration in the energy coupling between the magnetosphere and ionosphere. In Sect. 3.1, we note 
that three hours is a conservative estimate for the duration of the ULF pulsations observed on the 24-25 November 2001, as can be seen from the ionospheric velocity perturbations detailed in R05 Fig. 9; however, we do not have a sufficient amount of information to establish whether the pulsations are ubiquitously resonant. Figure 6 shows the unfiltered $\mathrm{H}$-component amplitude from the $\mathrm{CGO}$ magnetometer in Alaska for 00:00-10:00 UT on the 25 November 2001. Clearly evident from Fig. 6 is the longevity of the pulsation as the magnetometer rotates through the dusk sector. The frequency of these large-amplitude magnetic pulsations is constant and around $\sim 1.5 \mathrm{mHz}$ during the interval, although we do not have sufficient information to verify whether the ULF pulsation is resonant for the entire $8 \mathrm{~h}$ interval. However, if we extend the FLR interval in our analysis to $8 \mathrm{~h}$, as suggested by Fig. 6, the toroidal mode "Global" field line resonance would deposit $\sim 10^{15} \mathrm{~J}$ of energy into the resistive ionosphere, remarkably close to the values calculated by Østgaard et al. (2002b) in their substorm studies.

In Sect. 3.2, we present a comparison of the net fieldaligned Poynting vector and the ionospheric electric fields. Southwood and Hughes (1983) stated that the net downward Poynting vector must be balanced by Joule heating of the ionosphere in the steady state. In this interval, we observationally verify this; the net downward Poynting vector measured in the equatorial plane, and mapped down into the ionosphere is remarkably close to the point measurement of the ionospheric Joule heating rate. Previous studies have not had the luxury of conjugate satellite measurements with which to calibrate their estimates of Pedersen conductance (e.g., GW80; AP84; B05). We are therefore confident that our estimates of power and energy dissipation are as accurate as possible.

On a final note, Gla $\beta$ meier et al. (1999) note that:

"definitive proof of the existence of magnetospheric field line resonances requires detailed consideration of Poynting fluxes in the resonance region where isotropic energy flow is converted into field-aligned energy flow. Such an observation has not yet been reported and field line resonances need to be regarded as a well argued conjecture, but not as a proven phenomenon",

R05 presented the definitive existence of a toroidal mode FLR, by detailing magnetospheric observations of the electric and magnetic field structure of the FLR whilst the Polar spacecraft was conjugate to the "Churchill Line" of the CARISMA magnetometer array. In this study, we compare the field-aligned downgoing Poynting vector with point measurements of the ionospheric Joule heating.

\section{Conclusions}

We present an interval on the 25 November 2001 and previously reported in $\mathrm{R} 05$. In it, discrete frequency pulsations in the magnetosphere resulted from the excitation of a magnetospheric waveguide mode, perhaps excited via the Kelvin-Helmholtz instability and over-reflection at the duskside magnetopause. In this paper, we analyse the groundbased and ionospheric measurements more fully, to estimate the energy deposition into upper atmosphere via Joule heating. By comparison of in-situ electric and magnetic field measurements conjugate to ground-based magnetic measurements, we can verify the estimate of the Pedersen conductance obtained with the IRI conductance model. We find that the Pedersen conductance required to balance the net downward Poynting vector with the ionospheric Joule heating to be consistent with the predicted model IRI Pedersen conductance. Since $\Sigma_{p}$ is such a difficult ionospheric parameter to measure over large-scales we are at least confident that the point comparison is accurate.

Previous studies (e.g., GW80) have estimated the total energy deposition to be $\sim 10^{13} \mathrm{~J}$, or $4 \%$ of the total energy deposition of a small magnetospheric substorm. In this paper, we estimate that under favourable conditions and a global energisation of the magnetospheric waveguide, $10^{14}-10^{15} \mathrm{~J}$ of energy may be deposited into the ionosphere via Joule heating, an order of magnitude larger than the GW80 study, and $\sim 30 \%$ of the energy budget of a substorm cycle reported in $\varnothing 02 \mathrm{~b}$. Indeed, if the resonance interval is of a longer duration, the energy deposited in the ionosphere via field line resonance may be essentially equal to that deposited during a substorm cycle. As such, this study suggests that any global excitation of ULF waves in the magnetosphere must be taken into account when estimating the high-latitude and auroral energy budget.

Acknowledgements. The authors are indebted to M. Lester and K. H. Glaßmeier for helpful discussions. This work was funded by the Canadian Space Agency and the National Science and Engineering Research Council. We are grateful to the Geophysical Institute of the University of Alaska Fairbanks for providing the College International Geophysical Observatory magnetometer (CGO) magnetometer data.

Topical Editor M. Pinnock thanks F. Menk and another anonymous referee for their help in evaluating this paper.

\section{References}

Allan, W., White, S. P., and Poulter, E. M.: Impulse-excited hydromagnetic cavity and field line resonances in the magnetosphere, Planet. Space Sci., 34, 371-385, 1986.

Allan, W. and Poulter, E. M.: The spatial structure of different ULF pulsation types: a review of STARE radar results, Rev. Geophys., 22, 85-97, 1984.

Baddeley, L. J., Yeoman, T. K., Wright, D. M., Davies, J. A., Trattner, K. J., and Roeder, J. L.: Morning sector drift-bounce resonance driven ULF waves observed in artificially-induced HF radar backscatter, Ann. Geophys., 20, 1487-1498, 2002, http://www.ann-geophys.net/20/1487/2002/.

Baddeley, L. J., Yeoman, T. K., Wright, D. M., Trattner, K. J., and Kellet, B. J.: On the coupling between unstable magnetospheric 
particle populations and resonant high $\mathrm{m}$ ULF wave signatures in the ionosphere, Ann. Geophys., 23, 567-577, 2005

Bilitza, D.: International Reference Ionosphere 1990, NSSDC 9022, Greenbelt, Maryland, 1990.

Buchert, S. C., Fujii, R., and Glaßmeier, K.-H.: Ionospheric conductivity modulation in ULF pulsations, J. Geophys. Res., 104, 10 119-10 133, 1999.

Chen, L. and Hasegawa, A.: A theory of long-period magnetic pulsations, 1, Steady state excitation of field line resonance, J. Geophys. Res., 79, 1024-1032, 1974.

Coroniti, F. V. and Kennell, C. F.: Electron precipitation pulsations, J. Geophys. Res., 75, 1279-1289, 1970.

Dessler, A. J.: Ionospheric heating by hydromagnetic waves, J. Geophys. Res., 64, 397-401, 1959.

Dungey, J. W.: Electrodynamics of the outer atmosphere, Proceedings of the Ionosphere, Phys. Soc. Of London, London, p. 255, 1955.

Dungey, J. W.: Interplanetary Magnetic Field and the Auroral Zones, Phys. Rev. Lett., 6, 47-48, 1961.

Fenrich, F. R. and Samson, J. C.: Growth and Decay of field line resonances, J. Geophys. Res., 102, 20 031-20 039, 1997.

Fillingim, M. O., Parks, G. K., Chua, D., and Lin, R. P.: Comparison of plasma sheet and auroral electron energy fluxes during substorms, Proc. 6th International Conference on Substorms, University of Washington, 382-387, 2002.

Gla $\beta$ meier, K.-H., Volpers, H., and Baumjohann, W.: Ionospheric Joule Dissipation as a Damping Mechanism ofor High Latitude ULF Pulsations: Observational Evidence, Planet. Space Sci., 32, 1463-1466, 1984.

Gla $\beta$ meier, K.-H., Othmer, C., Cramm, R., Stellmacher, M., and Engebretson, M.: Magnetospheric Field Line Resonances: A Comparative Planetology Approach, Surv. Geophys., 20, 61109, 1999.

Greenwald, R. A. and Walker, A. D. M.: Energetics of long period resonant hydromagnetic waves, Geophys. Res. Lett., 7, 745-748, 1980.

Greenwald, R. A., Baker, K. B., Dudeney, J. R., Pinnock, M., Jones, T. B., Thomas, E. C., Villain, J.-P., Cerisier, J.-C., Senior, C., Hanuise, C., Hunsucker, R. D., Sofko, G., Koehler, J., Nielsen, E., Pellinen, R., Walker, A. D. M., Sato, N., and Yamagishi, H.: Darn/Superdarn: A global view of the dynamics of high-latitude convection, Space Sci. Rev., 71, 761-796, 1995.

Hasegawa, A.: Particle acceleration by MHD surface wave and formation of aurora, J. Geophys. Res., 81, 5083-5090, 1976

Hughes, W. J. and Southwood, D. J.: The screening of micropulsation signals by the atmosphere and ionosphere, J. Geophys. Res., 81, 3234-3240, 1976.

Jacobs, J. A., Kato, Y., Matsushita, S., and Troitskaya, V. A.: Classification of geomagnetic micropulsations, J. Geophys. Res., 69, 180-181, 1964.

Keiling, A, Wygant, J. R., Cattell, C. A., Mozer, F. S., and Russell, C. T.: The global morphology of wave Poynting flux: Powering the aurora, Science, 299, 383-386, 2003.

Kivelson, M. G. and Southwood, D. J.: Resonant ULF waves: A new interpretation, J. Geophys. Res., 12, 49-52, 1985.

Kivelson, M. G. and Southwood, D. J.: Coupling of global magnetospheric MHD eigenmodes to field line resonances, J. Geophys. Res., 91(10), 4345-4351, 1986.

Mann, I. R., Wright, A. N., Mills, K. J., and Nakariakov, V. M.: Ex- citation of magnetospheric waveguide modes by magnetosheath flows, J. Geophys. Res., 104, 333-353, 1999.

Mann, I. R., Voronkov, I., Dunlop, M., Donovan, E., Yeoman, T. K., Milling, D. K., Wild, J., Kauristie, K., Amm, O., Bale, S. D., Balogh, A., Viljanen, A., and Opgenoorth, H. J.: Co-ordinated ground-based and Cluster observations of large amplitude global magnetospheric oscillations during a fast solar wind speed interval, Ann. Geophys., 20, 405-425, 2002,

http://www.ann-geophys.net/20/405/2002/.

Milan S. E., Yeoman, T. K., Lester, M., Moen, J., and Sandholt, P. E.,

Post-noon two-minute period pulsating aurora and their relationship to the dayside convection pattern, Ann. Geophys., 17, 877891, 1999,

http://www.ann-geophys.net/17/877/1999/.

Milan, S. E., Sato, N., Masaki, E., and Moen, J.: Auroral forms and the field-aligned current structure associated with field line resonances. J. Geophys. Res., 106, 25 825-25 833, 2001.

Newton, R. S., Southwood, D. J., and Hughes, W. J.: Damping of geomagnetic pulsations by the ionosphere, Planet. Space Sci., 26, 201-209, 1978.

Østgaard, N., Vondrak, R. R., Gjerloev, J. W., and Germany, G.: A relation between the energy deposition by electron precipitation and geomagnetic indices during substorms, J. Geophys. Res., 107, 1246, doi:10.1029/2001JA002003, 2002a.

Østgaard, N., Germany, G. Stadsnes, J., and Vondrak, R. R.: Energy analysis of substorms based on remote sensing techniques, solar wind measurements and geomagnetic indices, J. Geophys. Res., 107, 1233, doi:10.1029/2001JA002002, 2002b.

Ozeke, L. G., Mann, I. R., and Mathews, J. T., The influence of asymmetric ionospheric Pedersen conductances on the field-aligned phase variation of guided toroidal and guided poloidal Alfvén waves, J. Geophys. Res., 110, A08210, doi:10.1029/2005JA011167, 2005.

Pokhotelov, O. A., Pilipenko, V. A., and Amata, E.: Drift anisotropy instability of a finite- $\beta$ magnetospheric plasma, Planet. Space Sci. 33, 1229-1241, 1985.

Rae, I. J., Donovan, E. F., Mann, I. R., Fenrich, F. R., Watt, C. E. J., Milling, D. K., Lester, M., Lavraud, B., Wild, J. A., Singer, H. J., Rème, H., and Balogh, A.: Evolution and Characteristics of Global Pc5 ULF Waves During a High Solar Wind Speed Interval., J. Geophys. Res., 110, A12211, doi:10.1029/2005JA011007, 2005.

Rae, I. J., Mann, I. R., Watt, C. E. J., Kistler, L. M., and Baumjohann, W.: Equator-S observations of drift-mirror mode waves in the dawn-side magnetosphere, J. Geophys. Res., 112, A11203, doi:10.1029/2006JA012064, 2007.

Rae, I. J., Mann, I. R., Dent, Z. C., Milling, D. K., Donovan, E. F., Spanswick, E.: Multiple Field Line Resonances: Optical, Magnetic and Absorption Signatures, Planet. Space Sci., 701-713, doi:10.1016/j.pss.2006.02.009, 2007.

Rostoker, G., Samson, J. C., Creutzberg, F., Hughes, T. J., McDiarmid, D. R., McNamara, A. G., Vallance Jones, A., Wallis, D. D., and Cogger, L. L.: CANOPUS - A ground based instrument array for remote sensing the high latitude ionosphere during the ISTP/GGS program, Space Sci. Rev., 71, 743-760, 1995.

Samson, J. C., Jacobs, J. A., and Rostoker, G., Latitude-dependent characteristics of long-period geomagnetic micropulsations. J. Geophys. Res., 76, 3675-3683, 1971. 
Samson, J. C., Hughes, T. J., Creutzberg, F., Wallis, D. D.: Greenwald, R. A., and Ruohoniemi, J. M., Observations of a detached, discrete arc in association with field line resonances, J. Geophys. Res., 96, 15 683-15 695, 1991.

Samson, J. C., Harrold, B. G., Ruohoniemi, J. M., and Walker, A. D. M.: Field line resonances associated with MHD waveguides in the magnetosphere, Geophys. Res. Lett., 19, 441-444, 1992.

Samson, J. C., Cogger, L. L., and Pao, Q.: Observations of field line resonances, auroral arcs, and auroral vortex structures, J. Geophys. Res., 101, 17 373-17383, 1996.

Southwood, D. J., Dungey, J. W., Etherington, R. J.: Bounce resonant interaction between pulsations and trapped particles, Planet Space Sci., 17, 349-361, 1969.

Southwood, D., J. and Hughes, W. J.: Theory of hydromagnetic waves in the magnetosphere, Space Sci. Rev., 35, 301-366, 1983.
Southwood, D. J.: Some features of field line resonances in the magnetosphere, Planet. Space Sci., 22, 483-491, 1974.

Walker, A. D. M., Greenwald, R. A., Stuart, W. F., and Green, C. A.: STARE auroral radar observations of Pc5 geomagnetic pulsations, J. Geophys. Res., 84, 3373-3388, 1979.

Woch, J., Kremser, G., Korth, A., Pokhotelov, O. A., Pilipenko, V. A., Nezlina, Y. M., and Amata, E.: Curvature-driven drift mirror instability in the magnetosphere, Planet. Space Sci., 36, 383393, 1988.

Wright, A. N.: Dispersion and wave coupling in inhomogenous MHD waveguides, J. Geophys. Res., 99, 159-167, 1994. 